Brazilian Journal

of Chemical

ISSN 0104-6632

Printed in Brazil

Engineering

www.scielo.br/bjce

Vol. 35, No. 02, pp. 615 - 622, April - June, 2018

dx.doi.org/10.1590/0104-6632.20180352s20170210

\title{
CONVECTIVE-DIFFUSIVE HEAT TRANSFER IN TUBES: ROLE OF THE HYDRODYNAMICS OF FLOW ON THE FEASIBILITY DOMAIN OF THE ASYMPTOTIC TEMPERATURE PROFILES
}

\author{
A. Nastasia Allred ${ }^{1}$, Yung-Way Liu ${ }^{2}$ and Pedro E. Arce ${ }^{1 *}$ \\ ${ }^{1}$ Department of Chemical Engineering, Tennessee Technological University, \\ Prescott 214, 1020 Stadium Drive, P. O. Box 5013, Cookeville, TN 38505, \\ Tennessee, USA \\ ${ }^{2}$ Department of Mathematics, Tennessee Technological University, Bruner Hall 302 , \\ P. O. Box 5054, Cookeville, TN 38505, Tennessee, USA
}

(Submitted: April 20, 2017; Accepted: June 14, 2017)

\begin{abstract}
In this contribution, an assessment of the role of the hydrodynamics of flow on the asymptotic solutions used to describe convective-diffusive heat transfer in packed-bed and capillary tubes of cylindrical geometry is reported. Such asymptotic solutions are intrinsically dependent from the type of hydrodynamics present in the tubes. They describe the temperature profiles for heat transfer problems found in packed-bed and capillary systems with axial convective and radial diffusive transport and having a constant heat flux at the outer tube wall. The role of the hydrodynamics of the flow on the asymptotic temperature profiles and their feasibility region of validity for both the packed-bed and capillary tube cases were assessed and feasibility ranges identified. A brief comparison of the predictions for the two cases analyzed is also included. The analysis of the impact of the hydrodynamics on the ranges of validity for the asymptotic temperature profiles seems to be first one available.
\end{abstract}

Keywords: asymptotic solution, temperature profile, mathematical modeling, convective-diffusive, feasibility region

\section{INTRODUCTION}

Convective-diffusive heat transfer in capillary and packed-bed tubes is relevant in a variety of process applications found in engineering practice. These include, for example, chemical reactors, industrial heaters, evaporators used in food processing, and thermal and energy storage units (Levenspiel, 1998; Bird et al, 2002; Arce and Trigatti, 1994). In this contribution, an analysis of capillary systems (with laminar flow of Newtonian fluids) and packed-bed tubes (with plug flow models) is reported for the case with cylindrical geometry. The focus of the analysis is on studying the role of the hydrodynamics of the flow on the range of feasibility (or domain associated with the radial and axial variables) of the asymptotic temperature solutions (valid for distances away from the entrance of the tube) for the convective-diffusive heat transfer problem. Both heating and cooling cases for the packed-bed tubes and capillary tubes can be

* Corresponding author: E-mail: PArce@tntech.edu 
studied; however, this contribution focuses solely on the cases of cooling. The asymptotic temperature profiles are based on the fact that convective-diffusive transfer within the domain is handled by a constant flux at the outer capillary boundary. The analysis of the role of the hydrodynamics on the asymptotic solutions is very important in predicting the temperature behavior, as well as in the design of chemical reactors, heat exchangers, etc. (Levenspiel, 1998; Whitaker, 1977). It appears that neither the effect of the hydrodynamics on the feasibility ranges nor the comparison between two different types of flows for the asymptotic solutions are reported in the literature to our best knowledge.

\section{PLUG FLOW HYDRODYNAMICS MODEL IN PACKED-BED TUBES.}

The convective-diffusive transfer model for the case of a plug flow in a packed-bed tube of cylindrical geometry and with constant flux at the external walls (see Figure 1) is based on the previous work by Arce and Trigatti (1994). The basic microscopic model with axially convective and radially diffusive heat transfer is given by:

$$
\rho_{f} c_{p} \cup \frac{\partial T}{\partial z}=\frac{k}{r} \frac{\partial}{\partial r}\left(r \frac{\partial T}{\partial r}\right)
$$

where $k$ is the thermal conductivity, $\rho_{\mathrm{f}}$ is the density of the fluid, $c_{p}$ is the heat capacity of the fluid, $v$ is the cross-sectional, uniform velocity of the fluid moving along the packed-bed housed in the cylindrical domain. With the assumptions stated above, the wall of the packed-bed tube at $\mathrm{R}_{\mathrm{c}}$ shows that the system (see Figure 1) either gains $(+)$ or loses $(-)$ heat at a constant flux, $\pm \mathrm{q}_{0}$; this situation leads to the fact that, at the center of the tube, there will be a maximum or minimum temperature value (Arce and Trigatti, 1994).

Therefore, for the "cooling case," the boundary conditions for equation (1) can be identified as: $\left.\frac{\partial T}{\partial r}\right|_{r=0}=0 \quad-\left.k \frac{\partial T}{\partial r}\right|_{r=R c}=q_{0} \quad T||_{z=0}=T_{0}$

When the following dimensionless variables (Bird et al., 2002) are chosen to be:

$\rho \equiv \frac{r}{R_{C}} \quad Z \equiv \frac{z k}{\rho_{f} c_{p} v R_{c}^{2}} \quad \theta \equiv \frac{T-T_{0}}{\frac{q_{0} R_{C}}{k}}$

Then, by using eq. (1) and eq. (3), the following non-dimensional microscopic model can be written as follows:

$$
\frac{\partial \theta}{\partial Z}=\frac{1}{\rho} \frac{\partial \theta}{\partial \rho}\left(\rho \frac{\partial \theta}{\partial \rho}\right)
$$

In addition, the boundary conditions as well as the condition at the entrance can be written, respectively, as:

$\left.\frac{\partial \theta}{\partial \rho}\right|_{\rho=0}=\left.0 \quad \frac{\partial \theta}{\partial \rho}\right|_{\rho=1}=-1 \quad \theta \mid z=0=0$

The asymptotic temperature solution, i.e., the solution valid for distances away from the entrance $(Z>0)$ for this differential model will be discussed in the paragraphs below.

\section{Asymptotic Temperature Solution:}

Asymptotic solutions of this type can be easily justified by using integral equations where the Green function is the kernel (Arce at al., 1988; Arce and Locke, 1994). Arce and Trigatti (1994) derived the asymptotic solution for the temperature profile in a packed-bed tube with convective-diffusive transport whose model was summarized in the section above. For the entire range of the radial variable $(0 \leq \rho \leq 1)$, such a solution is valid for distances away from the entrance (i.e., $\mathrm{Z}>0$ ) and it can be written as:

$$
\theta^{\infty}(\rho, Z)=C_{0} Z+\psi(\rho)
$$

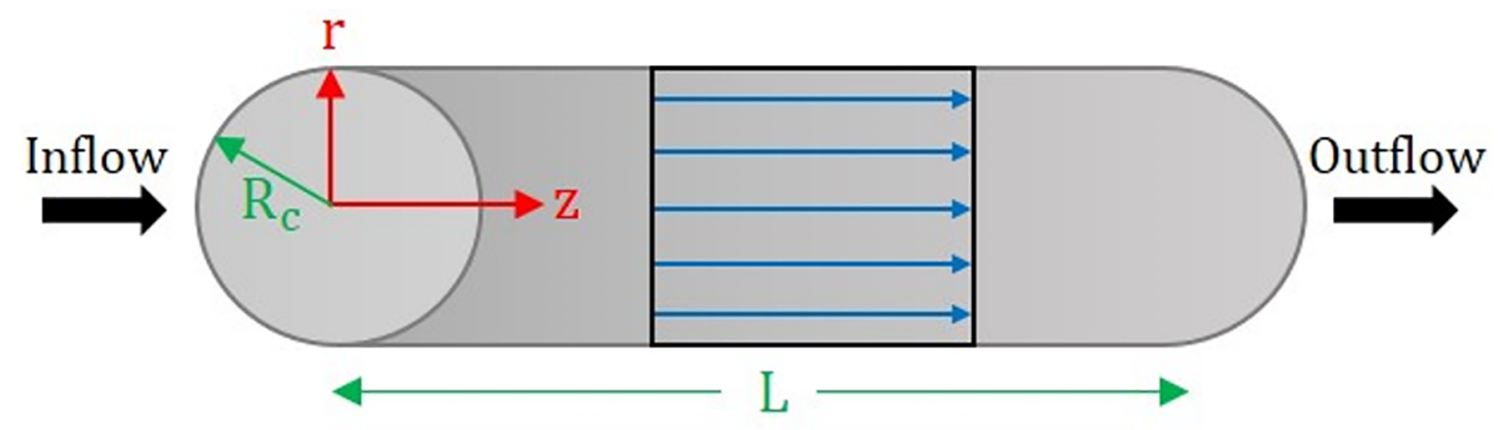

Figure 1. System Description of a Packed-Bed Tube. 
where $\mathrm{C}_{0}$ is a constant to be determined and the function $\psi(\rho)$ must satisfy the following differential equation:

$$
\frac{\partial}{\partial \rho}\left(\rho \frac{\partial}{\partial \rho} \psi(\rho)\right)=C_{0} \rho
$$

as well as the boundary conditions:

$$
\left.\frac{\partial \psi}{\partial \rho}\right|_{\rho=0}=\left.0 \quad \frac{\partial \psi}{\partial \rho}\right|_{\rho=1}=-1
$$

Since for the entire range of the radial variable $(0$ $\leq \rho \leq 1)$, the asymptotic temperature profile $\theta^{\infty}(\rho, Z)$ is valid for values of $Z>0$, the "entrance" condition to the capillary domain, at the inlet of the packed-bed (see Equation (2)), must be replaced by a macroscopicscaled condition valid also for the function $\theta^{\infty}(\rho, Z)$. This equation can be derived by using a "global" energy balance in the packed-capillary tube that, with non-dimensional variables, leads to (Arce and Trigatti, 1994):

$$
-Z=\int_{0}^{1} d \rho^{\prime} \rho^{\prime} \theta\left(\rho^{\prime}, Z\right)
$$

This condition leads to the following constrain for the function $\psi(\rho)$ :

$$
\int_{0}^{1} \rho^{\prime} \psi\left(\rho^{\prime}\right) d \rho^{\prime}=0
$$

This condition becomes useful to compute the "bulk" temperature of the system in order to validate the asymptotic solution. The solution for $\psi(\rho)$ from equation (7) leads to the following function:

$$
\psi(\rho)=\left(\frac{C_{0}}{4}\right) \rho^{2}+c_{1} \operatorname{Ln}(\rho)+c_{2}
$$

Constants $\mathrm{c}_{1}$ and $\mathrm{c}_{2}$ are readily determined by using the boundary conditions (8) in equation (11); function $\psi(\rho)$ is given by:

$$
\psi(\rho)=\frac{1}{4}\left(1-2 \rho^{2}\right)
$$

After the constant $\mathrm{C}_{0}$ was determined from equation (10) to give:

$$
C_{0}=2
$$

Finally, the asymptotic temperature profile, $\theta^{\infty}(\rho, Z)$ is given by:

$$
\theta^{\infty}(\rho, Z)=-2 Z+\frac{1}{4}\left(1-2 \rho^{2}\right)
$$

This asymptotic temperature profile is for the case of the packed-bed tube in cylindrical coordinates with the plug flow-like hydrodynamics. Since this solution is an asymptotic type of function, it requires both validation and ranges of validity. This task is performed in the section below.

\section{Validation and Feasibility Region of the Asymptot- ic Temperature Profile:}

The asymptotic temperature profile given by equation (14) requires both validation and a feasibility domain since it is valid for axial positions away from the entrance, i.e., $Z>0$. Arce and Trigatti (1994) reported a few points of validation for a solution such as this. First, they noted that the "bulk temperature" of the system is well satisfied by the asymptotic temperature profile as well as the "local" Nusselt number for the system, which in this case is $\mathrm{Nu}=8$. Furthermore, they also suggested that certain physical characteristics become handy for the validation of the asymptotic solution. For example, the smallest value of the axial variable must be identified in order to apply the temperature profile in the tube domain. Since, for the packed-bed tube, the system is assumed to be cooling, then, from the physical point of view, we can use a property associated with the function $\theta^{\infty}(\rho, Z)$. Arce and Trigatti (1994) noted that this function should remain within a given sign, i.e., negative since the system is cooling. However, it is possible that when $\mathrm{Z}$ approaches small values of the axial coordinate, the function $\theta^{\infty}(\rho, Z)$ could take positive values. More precisely, the function would tend to cross the axis $\theta^{\infty}(\rho, Z)$ since the validity of $\theta^{\infty}(\rho, Z)$ is for $Z>0$. Therefore, they suggested the following limiting condition for determining the feasibility of values for the independent variables $(\rho, Z)$ :

$$
\theta^{\infty}(\rho, Z) \equiv 0
$$

By using this condition, equation (14) can be written as:

$$
Z^{*}=\frac{1}{8}\left(1-2 \rho^{* 2}\right)
$$

where $Z^{*}$ and $\rho^{*}$ are all values that satisfy condition (15) that can be viewed as the boundary of feasible values of the asymptotic temperature profile. Note: The idea of a feasibility region is motivated by a contribution by Caram and Amundson (1977). Please note that equation (16) gives values for both $Z$ and $\rho$ and not just for the axial variable, $Z$, as it was thought 


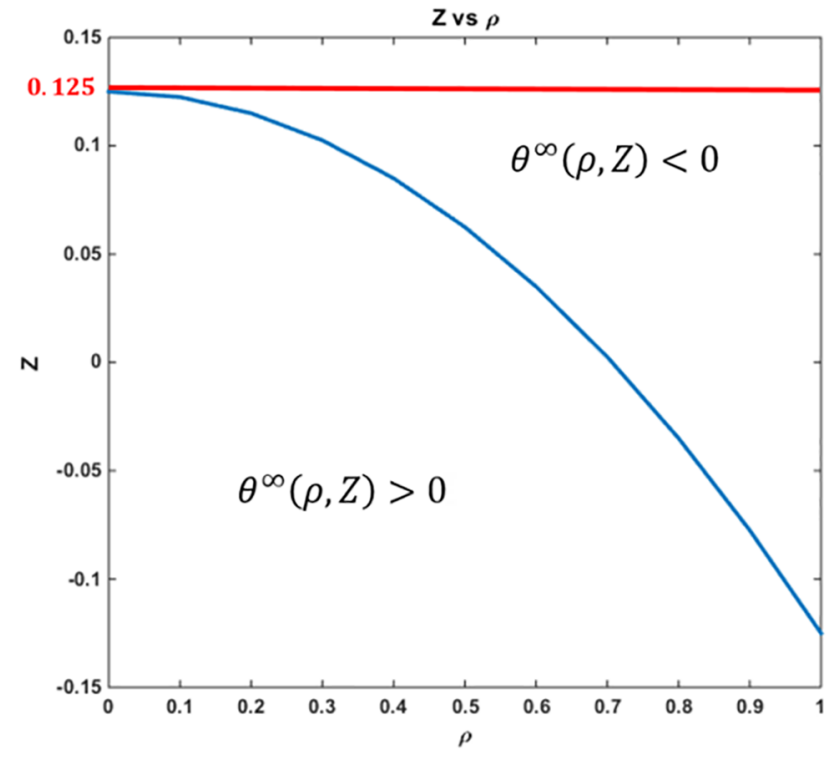

Figure 2. Feasibility Domain for Packed Bed Tube Flow System.

before. Moreover, Equation (16) may be used to sketch the graph that is presented in Figure 2. From this sketch, it is possible to see two regions, i.e.: 1the upper or physically meaningful domain $\left(\theta^{\infty}<0\right)$ and 2 - the bottom region where the temperature is not physically meaningful $\left(\theta^{\infty}>0\right)$ according to the physical constraints identified above. Furthermore, for $\rho \in 0 \leq \rho \leq 1$ and $Z<0.125$, the solution $\theta^{\infty}<0$ belongs to a physically meaningful domain. There is a region for values of $Z<0.125$ and for values of $\rho \epsilon$ $0 \leq \rho \leq 0.7071$ where both $Z$ and $\rho$ show values that are within the domain of the system; however, the values of the temperature within this region belong to the case where $\theta^{\infty}>0$ and, therefore, these are not physically meaningful. Alternatively, for the full domain of the radial variable $\rho \in 0 \leq \rho \leq 1$ to be considered, $Z$ is restricted to $Z<0.125$ in order for $\theta^{\infty}$ to be within the feasibility domain. On the other hand, if the full range of $Z \in 0 \leq Z \leq \infty$ must be taken into account, then only part of the domain for the radial variable can be used, i.e., $\rho \in 0.7071 \leq \rho \leq 1$ must be used in order for $\theta^{\infty}$ to be within the feasibility domain. These are limitations of the asymptotic temperature profile of the model and the values identified represent the complete domain of feasibility for both the radial as well as the axial variables based on the constraints originally suggested by Arce and Trigatti (1994).

\section{LAMINAR HYDRODYNAMIC MODEL FOR FLOWS IN CAPILLARY TUBES.}

The microscopic-scale model can be derived from the application of the energy equation with axially convective transport and with radially diffusive transport in a cylindrical capillary with laminar flow regime, i.e., the Poiseuille flow for Newtonian fluids (Whitaker, 1977; Bird, et al., 2002). The boundary conditions as well as the entrance condition associated with this microscopic model are similar to the previous case. For the cylindrical capillary tube (see Figure 3), the mathematical model for the steady state heat convective-diffusion equation under the general assumptions stated in the previous section, except for the flow regime that is now Poiseuille, is given by:

$$
\rho_{f} c_{p} \cup_{z}(r) \frac{\partial T}{\partial z}=\frac{k}{r} \frac{\partial}{\partial r}\left(r \frac{\partial T}{\partial r}\right)
$$

where the velocity profile, $v_{z}(r)$, is given by the usual parabolic shape in cylindrical coordinates (Bird et al., 2002):

$$
v_{Z}(r)=v_{Z}^{\max }\left(1-\left[\frac{r}{R}\right]^{2}\right)
$$

The boundary conditions are the same as in the previous case:

$$
\left.\frac{\partial T}{\partial r}\right|_{r=0}=0 \quad-\left.k \frac{\partial T}{\partial r}\right|_{r=R c}=q_{0}
$$

Please note that the boundary condition at the outer wall indicates a heating process that is taking place

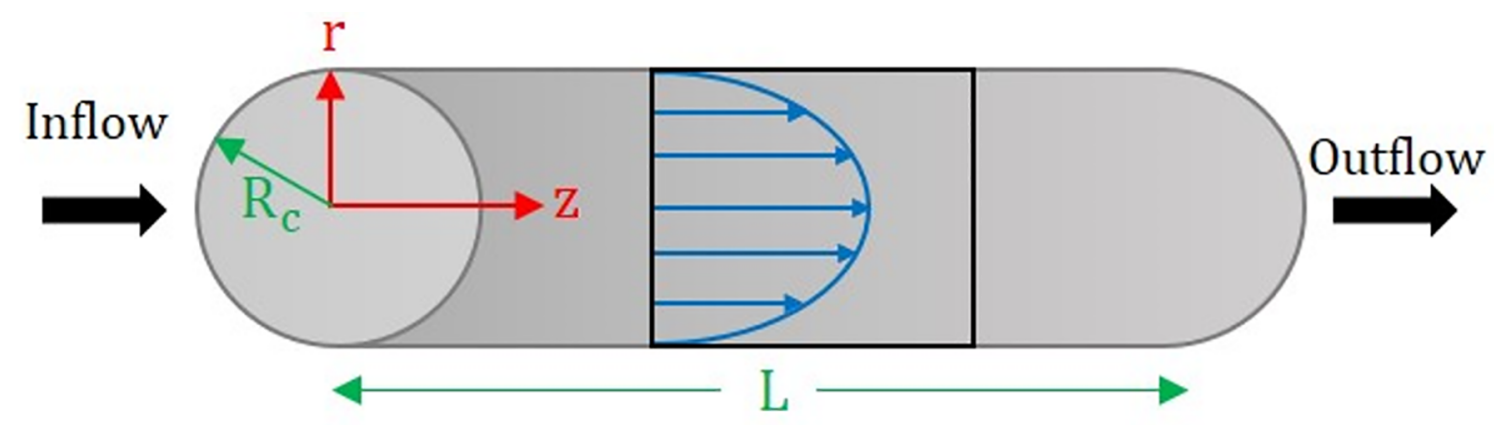

Figure 3. Description of a Capillary Tube Flow System. 
within the capillary. The entrance condition is given by specifying the temperature of the fluid at that location:

$$
T \mid z=0=T_{0}
$$

By defining the following dimensionless variables (Bird et al., 2002):

$$
\rho \equiv \frac{r}{R_{C}} \quad Z \equiv \frac{z k}{\rho_{f} c_{p} \nu_{Z}^{\max } R_{C}^{2}} \quad \theta \equiv \frac{T-T_{0}}{\frac{q_{0} R_{C}}{k}}
$$

The heat transfer microscopic model for the capillary domain with non-dimensional variables becomes the following:

$$
\left(1-\rho^{2}\right) \frac{\partial \theta}{\partial Z}=\frac{1}{\rho} \frac{\partial}{\partial \rho}\left(\rho \frac{\partial \theta}{\partial \rho}\right)
$$

The boundary conditions in equations (3) - (5) in non-dimensional terms are:

$$
\left.\frac{\partial \theta}{\partial \rho}\right|_{\rho=0}=\left.0 \quad \frac{\partial \theta}{\partial \rho}\right|_{\rho=1}=-\left.1 \quad \theta\right|_{z=0}=0
$$

An asymptotic temperature profile as the solution to this microscopic model will be discussed in the section below.

\section{Asymptotic Temperature Profile}

As before, for distances away from the entrance, i.e., $Z>0$, the temperature profile of the capillary domain can be approximated by a linear relation in the z-direction (Bird et al., 2002; Arce et al., 1988) superimposed on a non-linear function $\psi(\rho)$ to be determined. This assumption yields the mathematical results for the asymptotic temperature profile, $\theta^{\infty}(\rho, Z)$ that in terms of the nomenclature used previously leads to:

$$
\theta^{\infty}(\rho, Z)=C_{0} Z+\psi(\rho)
$$

The fact that this solution is valid for values of the axial distance such as $Z>0$ implies that the "entrance" condition at $\mathrm{Z}=0$ needs to be replaced by a "global energy balance" (Bird et al., 2002). The nomenclature $\theta^{\infty}(\rho, Z)$ has been used to indicate this characteristic of the general temperature profile, $\theta^{\infty}(\rho, Z)$. The global energy balance condition for the capillary under the conditions mentioned above is simply given by:

$$
2 \pi R_{C} q_{0} z=\int_{0}^{2 \pi} d \theta \int_{0}^{R_{C}} r d r \quad c_{p} \rho_{f} \mathcal{U}_{Z}(r)\left(T-T_{0}\right)
$$

Now, in dimensionless form, this equation leads to

$$
Z=\int_{0}^{1} \theta^{\infty}\left(\rho^{\prime}, Z\right)\left(1-\rho^{\prime 2}\right) \rho^{\prime} d \rho^{\prime}
$$

This condition is very helpful to write the following constraint for the function $\psi(\rho)$ in the following terms:

$$
\int_{0}^{1} d \rho^{\prime} \psi\left(\rho^{\prime}\right) \rho^{\prime}\left(1-\rho^{\prime 2}\right)=0
$$

In addition (Bird et al., 2002), function $\psi(\rho)$ must satisfy

$$
\frac{1}{\rho} \frac{d}{d \rho}\left(\rho \frac{d \psi}{d \rho}\right)=C_{0}\left(1-\rho^{2}\right)
$$

The boundary conditions associated with equation (28) are the following:

$$
\left.\frac{d \psi}{d \rho}\right|_{\rho=0}=\left.0 \quad \frac{d \psi}{d \rho}\right|_{\rho=1}=-1
$$

By solving equation (28) we arrive at:

$$
\frac{1}{\rho} \frac{d}{d \rho}\left(\rho \frac{d \psi}{d \rho}\right)=C_{0}\left(1-\rho^{2}\right)
$$
that

From the boundary conditions (29) it is concluded

$$
\begin{gathered}
c_{1}=0 \\
c_{2}=-4
\end{gathered}
$$

Inserting the values for the constants stated in (31) and (32) into equation (30), Equation (27) yields

$$
c_{2}=\frac{7}{24}
$$

which leads to the following solution for $\psi(\rho)$ :

$$
\psi(\rho)=\frac{\rho^{4}}{4}-\rho^{2}+\frac{7}{24}
$$

With equation (34) inserted into equation (24), the asymptotic solution is:

$$
\theta^{\infty}(\rho, Z)=-4 Z+\frac{\rho^{2}}{4}-\rho^{2}+\frac{7}{24}
$$

As indicated in the previous case for the packedbed tube, the solution for the temperature profile is of an asymptotic type and, therefore, validation and a domain of feasibility are needed. These tasks are performed in the section below. 


\section{Validation and Feasibility Domain of the Asymp- totic Temperature Profile:}

Equation (33) is an asymptotic function valid generally for $Z>0$ and, as such, it needs validation and the feasibility domain for the independent variables, $\rho$ and $\mathrm{Z}$. As in the previous case, the temperature profile can be used to determine the "bulk" temperature, $\theta_{\mathrm{b}}$ $(Z)$, in the capillary domain (Bird et al., 2002). This function turned out to be $\theta_{b}(Z)=-4 Z$. The linear portion of the asymptotic temperature profile is a direct calculation of the bulk temperature from the differential model (see equations (22) - (23)) and also yields exactly the same function, indicating a good validation of the asymptotic profile. In addition, the (local) Nusselt number can be calculated from the asymptotic solution and it produces the correct value for this case, $\mathrm{Nu}=4.364$ when the diameter is used as the characteristic length (Rolle, 2000).

As suggested by Arce and Trigatti (1994) and from the physical point of view, this system describes a cooling fluid under Poiseuille flow conditions. This implies that, according to the non-dimensional version of $\theta^{\infty}$ used here, the valid domain for the values of this solution should be $\theta^{\infty}<0$. In a similar way as for the packed-bed tube analyzed in the section above (see Section 2), values that are located within the domain of $\theta^{\infty}>0$ are not physically meaningful. As pointed out by Arce and Trigatti (1994), this potential behavior is because the validity of the asymptotic temperature profile for the axial variable is $\mathrm{Z}>0$. As in the previous case, the feasibility boundary between the two domains for $\theta^{\infty}$ can be calculated from the condition $\theta^{\infty}=0$ or alternatively:

$$
Z=\frac{1}{4}\left(\frac{\rho^{4}}{4}-\rho^{2}+\frac{7}{24}\right)
$$

From this function, one can infer that a graph of the function given in equation (34) will yield an upper region for the boundary between the two domains for $\theta^{\infty}$ that is the physical meaningful domain. (See Figure 4).

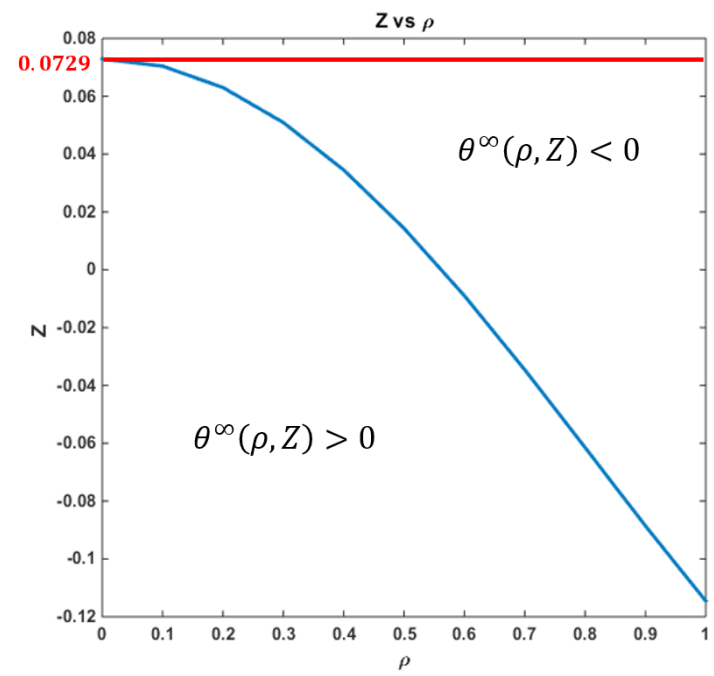

Figure 4. Feasibility Domain for Capillary Tube Flow System.

Also, from equation (34) the largest values of $Z$ for which $\theta^{\infty}>0$ is for the case of $\rho=0$; therefore, $Z=$ 0.0729 and the feasibility domain for the temperature profile (given by the asymptotic solution) will be for when $\mathrm{Z}>0.0729$ for the entire domain of the radial variable, $\rho \in 0 \leq \rho \leq 1$. Alternatively, for all values of the radial variable to be included, $\rho \in 0 \leq \rho \leq 1$ the smallest value of $Z$ when the solution $\theta^{\infty}$ is physically meaningful is $Z=0.0729$. Furthermore, if values of $Z$ are such that $Z<0.0729$, the solution is only valid for a portion of the radial variable, $\rho \in 0.54 \leq \rho \leq 1$. The reason behind this behavior (as indicated above) is the fact that $\theta^{\infty}(\rho, Z)$ changes sign when crossing the

Table 1. Comparative Values of the Predictions of the Asymptotic Solution with the Axial Variable

\begin{tabular}{lcc}
\hline Axial Distance $(Z)$ & $\boldsymbol{\theta}^{\infty}(\mathbf{0}, \boldsymbol{Z}) \mathrm{PFC}$ & $\boldsymbol{\theta}^{\infty}(\mathbf{0}, \boldsymbol{Z}) \mathrm{LFC}$ \\
\hline 0.01 & 0.230 & 0.252 \\
0.02 & 0.210 & 0.212 \\
0.03 & 0.190 & 0.172 \\
0.04 & 0.170 & 0.132 \\
0.05 & 0.150 & 0.092 \\
$0.0729^{*}$ & 0.104 & 0.000 \\
0.1 & 0.050 & -0.108 \\
$0.125^{*}$ & 0.000 & -0.208 \\
0.2 & -0.150 & -0.508 \\
0.3 & -0.350 & -0.908 \\
0.4 & -0.550 & -1.308 \\
0.5 & -0.750 & -1.708 \\
\hline
\end{tabular}


feasibility domain. This change of sign (in the values of the asymptotic solution) is a result for which the asymptotic solution is no longer physically meaningful since it has some limitations due to the approximation of a linear function with the axial variable, Z. However, the minimum value of $\mathrm{Z}$ is, in fact, very small and, from a practical point of view, the asymptotic solution becomes quite useful as a predictive tool for the temperature profile and for a large number of practical situations.

\section{DISCUSSION OF RESULTS AND BRIEF COMPARISON}

In the previous section, the analysis of the ranges of physically meaningful values related to the asymptotic solution for the Plug Flow Case (PFC) in a packedbed tube was discussed and illustrated and criteria for predicting the different values of the independent variables, $Z$ and $\rho$ (where the asymptotic solution is physically meaningful) were introduced. Likewise, the analysis for the Laminar Flow Case (LFC) in a cylindrical capillary tube was analyzed. In this section, an illustration of the different temperature values predicted by the criteria developed is included. In addition, a comparison of the potential impact of the hydrodynamics on the behaviors of the predictive values of the temperature for both cases is also performed.

In order to achieve these goals, Table 1 is constructed. This table illustrates the temperature values predicted by the asymptotic solutions for both the PFC and the LFC for different values of the axial variable, $Z$, and for the particular value of the radial variable of $\rho=0$. This value was selected since it is the most conservative value of the radial variable for changes in sign of the asymptotic solution since it is at the system wall $(\rho=1)$ where the cooling is taking place. Let us focus on the first and second column of Table 1, i.e., the PFC. Since this is a cooling case, $\theta^{\infty}(\rho, Z)<0$ in order to show physical significance. However, the values are all positive for the range $0<Z<0.125$. The first value of the temperature calculated for $Z>$ 0.125 is $Z=0.20$ and the value of $\theta^{\infty}(\rho, Z)$ at this point becomes negative $(-0.15)$. The trend continues to be with negative values (physically meaningful) for the temperature for all the values of $\mathrm{Z} \geq 0.2$ shown. This indicates an excellent consistence with the criteria that predicts that $Z=0.125$ is the critical point (please see the "*" in Table 1 on the value to indicate this condition).

The first and the last column in Table 1 indicate the behavior for the Laminar Flow Case (LFC). This system is also a cooling situation, and $\theta^{\infty}(\rho, Z)<0$ in order to be physically meaningful. However, all values of the axial variable such as $Z<0.0729$ produce positive values of the temperature. Values of the axial variable larger than $Z=0.0729$, see (for example) $Z=$ 0.1 yield negative values of the temperature $\theta^{\infty}(\rho, Z)=-$ 0.108 . Larger values of $Z$ (see Table 1 ) continue to yield negative values of the temperature. As before, this is an excellent confirmation that the value $\mathrm{Z}=$ 0.0729 is the critical value for changing the domain of physical significance of the asymptotic solution (we have indicated this with an "*").

It is interesting to note that the LFC shows a smaller value of the critical axial distance $(Z=$ 0.0729) compared to the PFC $(Z=0.125)$. In terms of validity, the LFC shows a larger domain of physically meaningful values in the axial variable than the PFC. This is a clear effect caused by the different hydrodynamic models associated with the two cases under analysis. These characteristics are in addition to the fact that the hydrodynamics yield different 'local' $\mathrm{Nu}$ numbers for the cases studied; the $\mathrm{Nu}$ for the case of the PFC is 3 and the one for the Poiseuille (LFC) case is $\mathrm{Nu}=8$. Both hydrodynamics lead to a linear bulk temperature with the axial coordinate, but the slopes of these lines are different as they reflect the effect of the non-linear function, $\psi(\rho)$.

\section{SUMMARY AND CONCLUDING RE- MARKS}

In this contribution, the effect of the hydrodynamic models that control the fluid velocity profile on the temperature profile of two cases of heat transfer in capillary tubes of cylindrical geometry is analyzed. Microscopic, differential models for two cases of heat transfer, i.e., a capillary system (with Poiseuille flow) under cooling conditions and a packed bed system (with plug flow) under cooling conditions were developed and their boundary conditions, formulated. Asymptotic solutions for the temperature profiles of both models were discussed for cases that are consistent with a constant wall flux of cooling at the outer boundary. Criteria for predicting the feasibility domains (of the independent variables, $\rho$ and $Z$ ) for the physically meaningful values of the temperature profiles have been reviewed and extended. These were first introduced by Arce and Trigatti (1994) and they now have been extended to include both the axial and the radial variables of the capillary domain, leading to a clear identification of a feasibility domain of the values of these variables for which the temperature profile 
shows physically meaningful values. The analysis suggests that, for these cases, the asymptotic solutions present limitations for both independent variables and not just for one of them as suggested before. It is interesting to point out that the technique used to yield the asymptotic temperature profiles utilizes a combination of microscopic models with macroscopic constraints that works efficiently to derive the proper asymptotic temperature function. The comparison table (see Table 1) for the values predicted for the feasibility domain indicates an important influence of the hydrodynamics of the flow. Finally, these solutions and the range of validity are useful to improve the understanding of the behavior of the systems described and to show the impact of the hydrodynamics on the asymptotic solutions for the temperature profiles.

\section{ACKNOWLEDGEMENTS}

An earlier effort related to this research was supported by a Fellowship to PA from the National Council of Research (CONICET), Argentina. A. Nastasia Allred is supported by a University Graduate Fellowship at Tennessee Technological University, Cookeville, TN-USA.

\section{NOMENCLATURE}

$c_{p}$ - Heat capacity of the cooling fluid

$C_{0}$ - Asymptotic solution constant

$c_{1}, c_{2}$ - Integration constants

$\mathrm{k}$ - Thermal conductivity of fluid

L - Length of tube

$\rho_{f}$ - Density

$q_{0}$ - Heat flux at the wall of the system

$r$ - Radial position

$\rho$ - Non-dimensional radial position

$R_{c}$ - Radius of tube

$\mathrm{T}$ - Temperature

$T_{0}$ - Temperature at $\mathrm{z}=0$

$\theta$ - Non-dimensional temperature

$\theta^{\infty}$ - Asymptotic and non-dimensional temperature

$v$ - Uniform velocity of the fluid in PFC $v_{z}^{\max }$ - Maximum velocity in LFC

$v_{z}(\mathrm{r})$ - Velocity of fluid in LFC

$\psi(\rho)$ - Asymptotic function in $\rho$

$\mathrm{z}$ - Axial position

Z - Non-dimensional axial position

$\mathrm{Nu}$ - Nusselt Number hL/k

$h$ - Convective heat transfer coefficient

\section{REFERENCES}

Arce, P. E. and Trigatti, I. M. B., Convective-Diffusive Heat Transfer in Packed-Bed with Constant Heat Flux at the Wall: An Asymptotic Solution for $\zeta \rightarrow$ Large. International Communications in Heat and Mass Transfer, 21(3), p. 435-446(1994)

Arce, P. E., Cassano, A. E., and Irazoqui, H. A., The Tubular Reactor with Laminar Flow Regime: An Integral Equation Approach-1. Homogeneous Reaction with Arbitrary Kinetics, Computers and Chemical Engineering, 12, p. 1103-1113 (1988)

Arce, P. E. and Locke, B. R., Transport and Reaction: An Integral Equation Approach. Mathematical Formulation, Computational Approaches, invited chapter in "Research Trends in Chemical Engineering," Menon J. (Ed.), Council of Scientific Research Integration, Vilayil Gardens, Trivansrum, India, vol 2, p. 89-158 (1994)

Bird, R. B., Stewart, W. E., and Lightfoot, E. N., Transport Phenomena, Second Ed., Wiley, New York, p. 311-316 (2002)

Caram, H. and Amundson, N. R., Diffusion and Reaction in a Stagnant Boundary Layer about a Carbon Particle, Ind. Eng. Chem. Fundamen., 16 (2), p. 171-181 (1977)

Levenspiel, O., Engineering Flow and Heat Exchange, Revised Edition, Plenum Press, New York, NY (1998)

Mansour, A., Sheboul, M., Hussein, A., and Nusayr, A., An analytical solution for diffusion and reaction in a laminar flow tubular reactor, International Communications in Heat and Mass Transfer, 16, p. 603-608 (1989)

Rolle, K. C., Heat and Mass Transfer, Prentice Hall, Upper Saddle River, New Jersey (2000)

Whitaker, S. Fundamental Principles of Heat Transfer, Pergamon Press, New York, NY (1977) 